\title{
EXCHANGE RATES FORECASTING: \\ CAN JUMP MODELS COMBINED WITH \\ MACROECONOMIC FUNDAMENTALS HELP?
}

\section{Tomáš Bunčák*}

\begin{abstract}
Connection between macroeconomic variables and foreign exchange (FX) rates evaluated in the context of out-of-sample forecasting is a well-known problem in economics. We propose a method that utilizes stochastic models based on jump processes (namely the normal inverse Gaussian and Meixner models), combines them with macroeconomic fundamentals, and using a moving (rolling or recursive) regularized estimation procedure produces forecasts of FX rates. These are compared to benchmark models, namely the direct forecast and the Gauss model forecast. Empirical out-of-sample experiments are performed on EUR/USD and USD/DKK currencies.
\end{abstract}

Keywords: exchange rates forecasting, jump processes, macroeconomic fundamentals, out-ofsample testing, cross-validation

JEL Classification: C46, C53, F37

\section{Introduction}

Surveying the published work, we may find a lot of evidence against usefulness of macroeconomic information for the prediction of foreign exchange $(F X)$ rates. This problem is sometimes labelled as the exchange rate disconnect puzzle and originates from Meese and Rogoff (1983). In the literature, mostly they conclude that even though the standard macroeconomic models are able to (at least partially) describe in-sample movement of FX rate, however, out-of-sample forecast is rarely better than a (no-change) forecast of a random walk (with or without drift). There is a vast amount of literature on this topic covering different methods of prediction, using various economic fundamentals, all applied on miscellaneous FX rates and time periods; for a good overview (and an exhaustive list of references) see the comprehensive study Rossi (2013) for instance. We try to provide a novel approach to forecasting of FX rates combining stochastic models with jumps, predictors based on standard macroeconomic models such as purchasing power parity, monetary fundamentals model, and Taylor-rule model, and techniques of regularized estimation with cross-validation. Although there is a literature about modelling of FX rates with jump models employed - Jiang (1998), Božović (2008), Busch et al. (2005), Nirei and Sushko (2011), Bates (1996), Nekhili et al. (2002), Maheu and McCurdy (2008) among others - to our knowledge, combining jump processes with macroeconomic information in order to predict FX rates seems to be new. Also note that we work with the infinite activity jump processes (namely the normal inverse Gaussian (NIG) and Meixner models), which is not a very commonplace practice in the literature concerning FX rates forecasting.

* Tomáš Bunčák, The Masaryk Institute of Advanced Studies, Czech Technical University in Prague, Prague, Czech Republic (tomas.buncak@cvut.cz).

Author's work is supported by the Grant No. SGS13/163/OHK5/2T/32 from the Grant Agency of the Czech Technical University in Prague. 
Stochastic models incorporating jumps are quite appropriate for modelling of (highfrequency) returns of financial assets (in particular FX rates), see Bunčák (2013) for instance. Thus once we are provided with the parameters of such a model, we are capable of calculating various measures describing returns of the underlying financial series, say FX rate. We may easily calculate $d r i f t$ (the mean of returns), volatility (the variance of returns), and other characteristics such as skewness and kurtosis as well. Of course, if we know exact parameters of a jump model for a future period, we would know precise estimate of the future FX rate. Hence there is a question whether we would be able to predict these parameters so that the implied forecast of FX rate would become more accurate than a direct forecast using the same prediction procedure.

This idea can be also viewed from another perspective. Considering time series of calibrated parameters of a jump model in a range of subsequent periods, we may say we get a kind of filtered information that might be connected with macroeconomic variables in a different way than the FX rate itself. Consequently, this may (or may not) provide a better (out-of-sample) forecast of the FX rate. Motivated by this idea, we employ several measures evaluating forecast accuracy to compare our method of prediction with benchmarks derived from a rather standard way of FX rates forecasting.

The paper is organized as follows. Subsection 2.1 introduces macroeconomic predictors we use. Subsection 2.2 explains the role of jump models in the prediction of FX rates we undertake. Next Subsection 2.3 outlines benchmark models that are compared to the jump models framework. Our estimation procedure is clarified in Subsection 2.4. Forecast evaluation criteria are stated in Subsection 2.5. Finally, empirical test results are given in Section 3. All is concluded in Section 4.

\section{Forecasting Framework Description}

\subsection{Regressors derived from macroeconomic models}

In our modelling framework, we use predictors that are based on some of the well-known macroeconomic models, namely purchasing power parity, monetary fundamentals model, and Taylor-rule model. The basic idea of regressors construction is that each of these models gives an expression for a fundamental value of a chosen FX rate. Denoting logarithm of this value as $\tilde{s}_{t}$ for a time $t \geq 0$, we use predictors defined as $\tilde{s}_{t}-s_{t}$, where $s_{t}=\log \left(S_{t}\right)$ is the logarithm of the (nominal) FX rate process $S_{t}$ (units of home currency per one unit of foreign currency). Hence a predictive model might be used in the form

$$
\Delta s_{t+h \mid t}=s_{t+h}-s_{t}=a+b\left(\tilde{s}_{t}-s_{t}\right)+\varepsilon_{t}, t \geq 0, h>0 .
$$

Essentially, this is what we call a direct forecast, but we will elaborate upon this further. Note that these regressors will be also used for forecasting of jump model parameters, see Subsection 2.2. This design of predictors is not new and can be found in Engel et al. (2007) or Li et al. (2015), among others. Let us describe the used predictors in more detail now.

\subsubsection{Purchasing power parity}

Purchasing power parity (PPP) is a well-known concept in economics. It says that should two economies' purchasing powers be in parity, real exchange rate would be equal to one, i.e. two national price levels would be equal after currency adjustment. Expressing that with formulas we get 


$$
S_{t}^{r}=\frac{P_{t}^{*} S_{t}}{P_{t}}=1, t \geq 0
$$

where $S_{t}^{r}$ denotes the real exchange rate, $P_{t}^{*}$ is the foreign ${ }^{1}$ price level, and $P_{t}$ the domestic price level. Hence, taking logarithms gives us a fundamental (logarithmic) FX rate value implied by PPP, namely

$$
\tilde{s}_{t}=\log \left(P_{t}\right)-\log \left(P_{t}^{*}\right)=p_{t}-p_{t}^{*}, t \geq 0,
$$

which in turn yields our first regressor

$$
x_{t}^{1}=p_{t}-p_{t}^{*}-s_{t}, t \geq 0 .
$$

Of course, there is a lot of discussion about the PPP model itself, interested reader is referred to Rossi (2013) - as for any other regressor we introduce.

\subsubsection{Monetary fundamentals}

The second of our regressors is based on monetary fundamentals. Considering a model for (the logarithm of) real money

$$
m_{t}-p_{t}=-\eta i_{t}+\phi y_{t}, t \geq 0
$$

Where $m_{t}$ is the logarithm of the nominal money supply, $i_{t}$ denotes the nominal interest rate, and $y_{t}$ stands for the logarithm of the real output. Assuming that the same equation (with the same coefficients) holds for the foreign country and combining with Equation 1 we get a fundamental FX rate of the form

$$
\tilde{s}_{t}=\eta\left(i_{t}-i_{t}^{*}\right)-\phi\left(y_{t}-y_{t}^{*}\right)+m_{t}-m_{t}^{*}, t \geq 0 .
$$

As one can see in Bruyn et al. (2013) for instance, this can be simplified to the basic form of the monetary model where $\eta=0$ and $\phi=1$. That gives us the second predictor

$$
x_{t}^{2}=m_{t}-m_{t}^{*}-\left(y_{t}-y_{t}^{*}\right)-s_{t}, t \geq 0 \text {. }
$$

\subsubsection{Taylor rule predictor}

The last predictor we use is motivated by the (symmetric) Taylor rule. It is constructed in the following way. Consider a reaction function by which a central bank sets an interest rate policy

$$
i_{t}=c+c_{s^{r}} s_{t}^{r}+c_{\pi} \pi_{t}+c_{y} y_{t}^{g}+c_{i} i_{t-\Delta t}, t \geq 0
$$

Where $i_{t}$ is the domestic interest rate, $s_{t}^{r}=s_{t}+p_{t}^{*}-p_{t}$ is the logarithm of the real exchange rate, $\pi_{t}$ denotes the domestic inflation rate, $y_{t}^{g}$ is the logarithm of the domestic output gap. Now assume that a similar equation holds for the foreign interest rate, namely

$$
i_{t}^{*}=c^{*}+c^{*} s_{t}^{r^{*}}+c^{*}{ }_{t}^{*}+c_{y}^{*} y_{t}^{g^{*}}+c_{i}^{*} i_{t-\Delta t}^{*}, t \geq 0
$$

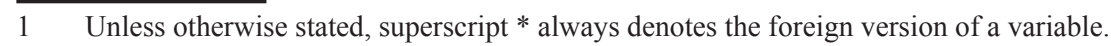


where superscript * denotes the foreign counterparts of variables or coefficients. Note that $s_{t}^{r^{*}}=-s_{t}^{r}, t \geq 0$. Recalling the well-known concept of uncovered interest rate parity we write

$$
\mathbb{E} s_{t+\Delta t}-s_{t}=i_{t}-i_{t}^{*}, t \geq 0
$$

where $\mathbb{E}$ denotes the expectation. If we plug (4) and (5) in (6) and assume that the coefficients for the domestic and foreign economy are equal (symmetric rule), we get an expression for the fundamental ${ }^{2} \mathrm{FX}$ rate

$$
\tilde{s}_{t}=c_{\pi}\left(\pi_{t}-\pi_{t}^{*}\right)+c_{y}\left(y_{t}^{g}-y_{t}^{g^{*}}\right)+2 c_{s^{r}}\left(s_{t}+p_{t}^{*}-p_{t}\right)+s_{t}, t \geq 0,
$$

where we have furthermore set $c_{i}=0$. Hence, in accord with our methodology, selection of specific values for the coefficients yields directly the regressor of the form

$$
x_{t}^{3}=1.5\left(\pi_{t}-\pi_{t}^{*}\right)+0.1\left(y_{t}^{g}-y_{t}^{g^{*}}\right)+0.1\left(s_{t}+p_{t}^{*}-p_{t}\right), t \geq 0 .
$$

Use of these coefficients is standard in the literature, as is advocated in Li et al. (2015) or Engel et al. (2007) for instance.

\subsection{The role of jump models}

We want to generate FX rate forecasts using jump models combined with macroeconomic fundamentals. To achieve this, we follow the next scheme:

1. We estimate (calibrate) parameters of a selected jump model in subsequent periods using log-returns data. ${ }^{3}$

2. Having (multivariate) time series of the calibrated jump model parameters, we apply moving (rolling or recursive) window estimation to get forecasts of these parameters. It means that for each of the jump model parameters we have a procedure that gives us the parameter prediction. In this step we use the macroeconomic information.

3. Once we are provided with the predictions of the jump model parameters, we generate implied forecasts of the FX rate.

Let us describe these steps in more detail now. As we mentioned before, we calibrate jump models on high-frequency (specifically one-hour) log-returns (or simply returns) that are defined as $r_{t}=s_{t}-s_{t-\Delta t}$ where $s_{t}=\log \left(S_{t}\right)$ is the logarithm of the FX rate process $S_{t}$. More concretely, we divide any given time period $\left[T_{1}, T_{2}\right]$ into smaller periods with time step $\Delta t>0$ getting time division $t_{i}=T_{1}+i \Delta t, i=0, \ldots, \frac{T_{2}-T_{1}}{\Delta t} .{ }^{4}$ Then we compute log-returns over these time steps. Using the log-returns data, we calibrate the selected jump model (i.e. we estimate its parameters). This is repeated for subsequent (non-overlapping) time periods so we get a multivariate time series of the jump model parameters evolving in time

2 Or here we may rather say expected.

3 Standardly, by calibration we mean selection (estimation) of parameters so that the theoretical distribution implied by the jump model is close to the empirical distribution of the log-returns. More about calibration can be found in Bunčák (2013) for instance.

4 Here any rounding is neglected for simplicity. 
(one set of the calibrated parameters for a period forms one observation of this multivariate time series). Summing up, the sequential calibration procedure follows these steps:

1. We divide the whole time period at our disposal $[0, T], T>0$ into smaller non-overlapping periods of length $h>0$, so we get intervals $I_{t, h}=[t, t+h], t=0, \ldots, T-h .^{5}$

2. In each subperiod $I_{t, h}$, we estimate the jump model parameters using observations of log-returns over shorter periods of length $0<\Delta t<<h{ }^{6}$ So for each $I_{t, h}$ we have the calibrated parameters (of a chosen jump model) denoted as $\boldsymbol{\theta}_{t, h}=\left(\theta_{t, h}^{1}, \ldots, \theta_{t, h}^{N}\right)^{\mathrm{T}}$, where $N$ is the number of parameters of the jump model; ${ }^{\mathrm{T}}$ denotes transposition (so vectors are columns standardly).

3. We end with a (multivariate) time series of parameters $\boldsymbol{\theta}_{t, h}, t=0, \ldots, T-h$.

Next we need to specify techniques used for the jump model parameters forecasting. We try to model each of the jump model parameters individually. There are, say, two groups of jump model parameters. One is the group that determines drift of the underlying stochastic process (i.e. location or shape parameters), the other is the group that influences volatility of the model process (i.e. scale parameters). We model the former using the information from the macroeconomic variables, the latter without the information using an autoregressive (AR) model only.

Hence we split the set of parameter indices into $R \cup A=\{1, \ldots, N\}$, where $R$ denotes indices predicted by regression, $A$ by an AR model. Let us first focus on the group labelled by $R$. Collecting the regressors (2), (3), and (7), we create a vector $\mathbf{x}_{t}=\left(x_{t}^{1}, x_{t}^{2}, x_{t}^{3}\right)^{\mathrm{T}}, t \geq 0$. Then we model each $\theta_{t, h}^{i}, i \in R$ by

$$
\theta_{t, h}^{i}=\kappa_{i}+\psi_{i}^{\mathrm{T}} \mathbf{x}_{t}+\varepsilon_{t}, t=0, \ldots, T-h,
$$

where $\kappa_{i} \in \mathbb{R} \quad \psi_{i} \in \mathbb{R}^{3}$ are estimated employing regularization as we will describe below. Since in (8) we use multiple macroeconomic predictors at once, we may call it a kitchensink regression as in Li et al. (2015) for instance. Considering the AR modelled parameters, for any $\theta_{t, h}^{j}, j \in A$ there is

$$
\theta_{t, h}^{j}=\gamma_{j}+\delta_{j} \theta_{t-h, h}^{j}+\varepsilon_{t}, t=h, \ldots, T-h,
$$

where $\gamma_{j} \in \mathbb{R}$ and $\delta_{j} \in \mathbb{R}$ represent parameters of the simple AR(1) model. Coefficients of these models are estimated in moving windows as we will describe later. Note that by $\varepsilon_{t}$ we denote an error term (residual) which is assumed to be a stochastic process with no serial correlation; and it is a different instance for each of the equations we state henceforth (even though we use the same symbol everywhere - for the simplicity of presentation). We tested for autocorrelation in all of the error terms that appear in our modelling and it seems to be no issue. In (8) or (9) we take no further assumptions on the residuals since we do not make any statistical inference based on these residuals directly and the estimation techniques we apply do not require any special assumptions here. However, should some additional assumptions (e.g. normality) be relevant at some point (such as the application

5 Time $t$ iterate with steps of length $h$; any rounding neglected for simplicity, overlapping in boundary points likewise.

6 Step $\Delta t$ shall be chosen so that we have enough log-return observations to calibrate the selected jump model in any period of length $h$. 
of the Evans-Lyons test later), we apply a few procedures (such as Q-Q plots or pertinent statistical tests) to inquire about their validity.

Finally, once we have estimated parameters of a jump model, we need to specify a way in which FX rate forecast is created. In fact, we try to predict log-return of an FX rate over $I_{t, h}$ at time $t$, so $h$ is the forecast horizon. Simply, FX rate forecasts are calculated as the mean of the jump model distribution with the corresponding estimated parameters. Let us specify this for our selected jump models.

\subsubsection{NIG model}

This model was introduced to finance in Barndorff-Nielsen (1997). It is a member of the class of generalized hyperbolic models, i.e. models based on processes constructed by Brownian motion subordination with the generalized inverse Gaussian subordinator. For more information about this class see Cont and Tankov (2003), Subsection 4.6.

NIG model has four parameters, as it common we denote them $\mu \in \mathbb{R}, \alpha>0, \delta>0$ and $0<|\beta|<\alpha$. Parameter $\alpha$ adjusts tail behaviour, that is steepness of the NIG distribution - the larger $\alpha$ is, the lighter are the tails of the distribution. Parameter $\beta$ controls the skewness of the distribution; $\beta<0$ produces left-skewed densities, $\beta>0$ right-skewed. Standardly, $\mu$ is the location (or drift) parameter. Finally, $\delta$ represents a measure of the volatility of returns; higher $\delta$ leads to more volatile returns. Considering these properties, we take $\mu$ and $\beta$ as the directional parameters modelled by the regression procedure (8), and $\delta, \sigma$ as the volatility parameters modelled by the AR model (9). Although we link only $\mu$ and $\beta$ to the macroeconomic predictors, we will see that the other parameters influence the FX rate log-return estimate as well.

This model provides a closed-form (probability) density for the returns, what allows us to use maximum likelihood estimation (MLE) for calibration. The density is of the form

$$
f_{r, \Delta t}(x)=\frac{\alpha \delta \Delta t}{\pi} \exp (\delta \gamma \Delta t+\beta(x-\mu \Delta t)) \frac{K_{1}\left(\alpha \sqrt{\delta^{2}(\Delta t)^{2}+(x-\mu \Delta t)^{2}}\right)}{\sqrt{\delta^{2}(\Delta t)^{2}+(x-\mu \Delta t)^{2}}}, x \in \mathbb{R}
$$

Where $K_{u}$ denotes the modified Bessel function of the second kind and index $u$, see Abramowitz and Stegun (1972) for example. Consider we have estimated parameters corresponding to log-returns over a unit of time, denote them $\boldsymbol{\theta}=(\alpha, \beta, \mu, \delta)^{\mathrm{T}}$. Then using the NIG model distribution of returns over any time $\Delta t>0$ (by the infinite divisibility of the Lévy processes) we have

$$
\mathcal{M}_{r}(\theta, \Delta t)=\mathbb{E} \Delta s_{t+\Delta t \mid t}=\left(\mu+\frac{\beta \delta}{\gamma}\right) \Delta t, \quad \mathcal{V}_{r}(\theta, \Delta t)=\operatorname{var}\left(\Delta s_{t+\Delta t \mid t}\right)=\frac{\delta \alpha^{2}}{\gamma^{3}} \Delta t,
$$

for the mean $\mathcal{M}_{r}$ and the variance $\mathcal{V}_{r}$ of returns, namely; $\gamma=\sqrt{\alpha^{2}-\beta^{2}}$. So if we have estimates of parameters $\hat{\boldsymbol{\theta}}_{t, h}=\left(\hat{\alpha}_{t, h}, \hat{\beta}_{t, h}, \hat{\mu}_{t, h}, \hat{\delta}_{t, h}\right)^{\mathrm{T}}$ for a future period $I_{t, h}=[t, t+h]$ at time $t$, we produce the estimate of FX rate log-return over this period by

$$
\Delta \hat{s}_{t+h \mid t}=\hat{s}_{t+h}-s_{t}=\mathcal{M}_{r}\left(\hat{\theta}_{t, h}, h\right),
$$

where $\mathcal{M}_{r}$ is from (10). Application of the formula for the variance will be described later (as a part of a simple out-of-sample trading test we carry out). The calibrated model parameters are illustrated in Figure 1. 


\subsubsection{Meixner model}

Figure 1 | NIG Model Parameters Corresponding to Hourly Log-Returns Fitted in Monthly Periods (EUR/USD rate, period 01/2004 - 12/2013)
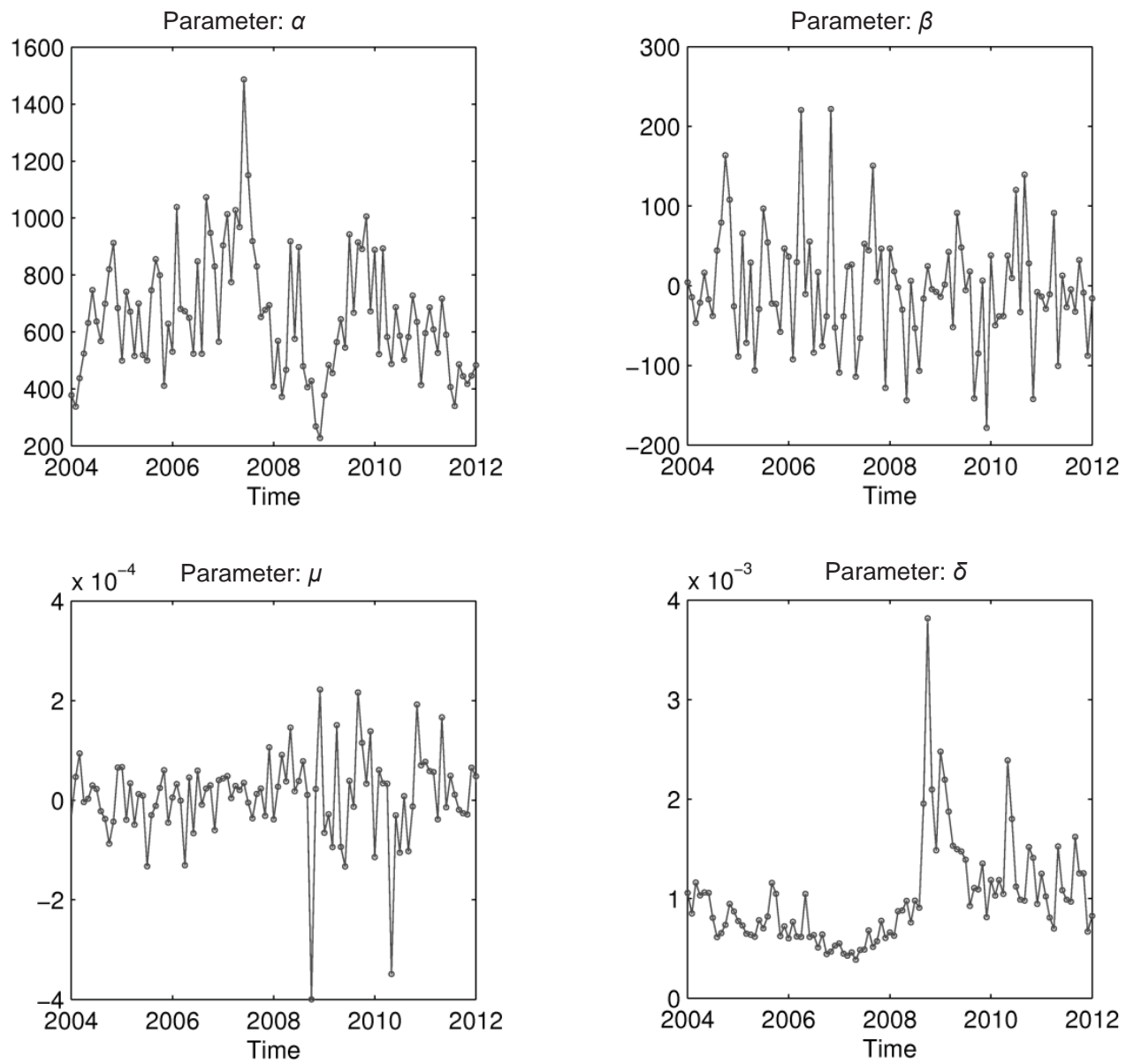

Source: own computation

Another example of the infinite activity Lévy models is the Meixner (MXN) model which was introduced to finance in Schoutens and Teugels (1998). This model also provides a density for the returns in a closed form, so we exploit this and use MLE for the calibration again. It has four parameters denoted as $\alpha>0,-\pi<\beta<\pi, \delta>0$, and $\mu \in \mathbb{R}$. ${ }^{7}$ Note that $\mu$ is the drift (or the location of returns) parameter, $\alpha$ controls scale of returns, $\beta$ and $\delta$ determine shape of the distribution - skewness and kurtosis in particular. The MXN model density is in the shape

7 Although we use the same notation as for the NIG model parameters, this shall not be confusing in our text and it emphasizes that the role of some parameters is comparable for both of the models. 


$$
f_{r, \Delta t}(x)=\frac{(2 \cos (\beta / 2))^{2 \delta \Delta t}}{2 \alpha \pi \Gamma(2 \delta \Delta t)} \exp \left(\frac{\beta(x-\mu \Delta t)}{\alpha}\right)\left|\Gamma\left(\delta \Delta t+\frac{i(x-\mu \Delta t)}{\alpha}\right)\right|^{2}, x \in \mathbb{R},
$$

where $\Gamma$ denotes the gamma function - see Abramowitz and Stegun (1972) if needed. The mean and the variance of the MXN model FX rate returns are given by

$$
\mathcal{M}_{r}(\boldsymbol{\theta}, \Delta t)=(\mu+\alpha \delta \tan (\beta / 2)) \Delta t, \quad \mathcal{V}_{r}(\boldsymbol{\theta}, \Delta t)=\frac{\alpha^{2} \delta}{\cos \beta+1} \Delta t,
$$

where again $\boldsymbol{\theta}=(\alpha, \beta, \mu, \delta)^{\mathrm{T}}$. For this model, $\mu$ and $\beta$ are modelled with the assistance of macroeconomic information by (8), $\alpha$ and $\delta$ with the AR model (9). Analogously to the NIG case, we predict by (11) with $\mathcal{M}_{r}$ from (12). MXN parameters evolving in time are demonstrated in Figure 2.

Figure 2 | MXN Model Parameters Corresponding to Hourly Log-Returns Fitted in Monthly Periods (EUR/USD rate, period 01/2004 - 12/2013)
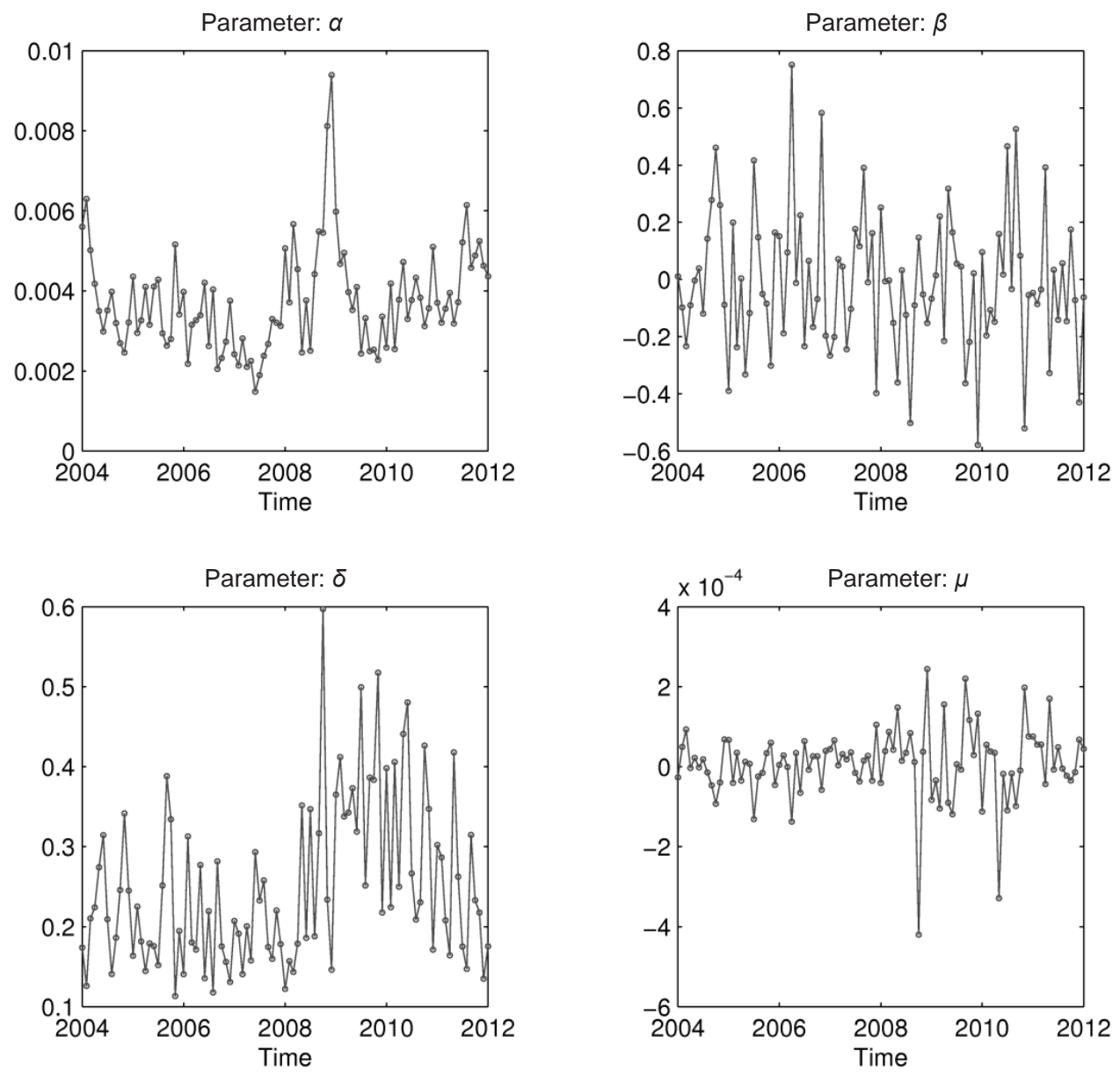

Source: own computation 


\subsection{Benchmarks}

\subsubsection{Gauss model prediction}

To get a benchmark, we follow the same procedure as for the jump processes but with returns modelled with the usage of the (continuous) Gauss (normal) distribution - i.e. the geometric Brownian motion model. For this model we have $\boldsymbol{\theta}=(\mu, \sigma)^{\mathrm{T}}$ where $\mu \in \mathbb{R}$ denotes the mean of the fitted log-returns and $\sigma>0$ represents the standard deviation. Since $\Delta s_{t+\Delta t \mid t} \sim \mathcal{N}\left(\mu \Delta t, \sigma^{2} \Delta t\right)$ we have

$$
\mathcal{M}_{r}(\theta, \Delta t)=\mu \Delta t, \quad \mathcal{V}_{r}(\theta, \Delta t)=\sigma^{2} \Delta t .
$$

The model parameters are calibrated straightforwardly by the well-known MLE formulas (in fact the sample mean and sample variance). Parameter $\mu$ is predicted using the economic predictors (8) and forecast of $\sigma$ is generated by the AR process (9). Projection of the FX rate return is gained by (11) with $\mathcal{M}_{r}$ from (13) plugged in.

\subsubsection{Direct forecast}

As was outlined in Subsection 2.1, in order to get a comparison with the jump model prediction, we also provide results for a direct FX rate returns forecast. That means modelling the log-returns directly by

$$
\Delta s_{t+h \mid t}=s_{t+h}-s_{t}=a+\mathbf{b}^{\mathrm{T}} \mathbf{x}_{t}+\varepsilon_{t}, t=0, \ldots, T-h .
$$

Estimation of the coefficients $\left(\alpha \in \mathbb{R}, \mathbf{b} \in \mathbb{R}^{3}\right)$ runs in the same way as for the regression model (8) of the jump model parameters - employing the regularized moving window estimation. This procedure is described in the next part.

\subsection{Moving regularized estimation with cross-validation}

Considering out-of-sample forecasting, the procedure of rolling or recursive regression estimation is not new. For example, it has been used in Li et al. (2015) and Rossi (2013) states many other references where one can find this method in a certain form. We follow a process which is very close to $\mathrm{Li}$ et al. (2015) and consists of these steps:

1. Consider we have data corresponding to a time period $[0, T], T>0$. We choose a point in time from which we start the procedure, say $\tau \in[0, T-h]$. We iterate over $t=\tau, \ldots, T-h$ with a chosen time step $h>0$.

2. For a step $t$, estimate coefficients of (8), (9), and (14) using data in $[0, t]$ (recursive version) or $[t-\tau, t]$ (rolling window version).

3. With the estimated coefficients, we produce forecasts of $\hat{\boldsymbol{\theta}}_{t, h}$ with the jump model derived FX rate prediction (11), then the Gauss model prediction, and also the direct forecast given by (14).

4. We keep the predicted FX rates and continue with $t:=t+h$ from step 2. If $t=T-h$, cease the procedure.

Of course, crucial is the second step in which we estimate coefficients of the models. Here we employ regularized estimators given by the so-called elastic net procedure, see 
Zou and Hastie (2005) for instance. The idea is that regularized estimators should identify the important predictors and have lower predictive errors than ordinary least squares estimates. Hence for the model (8) the estimated coefficients (using the data in period $[0, t])$ are given as a solution to

$$
\min _{\kappa_{i}, \psi_{i}}\left(\frac{h}{t} \sum_{s=0}^{t-h}\left(\theta_{s, h}^{i}-\kappa_{i}-\psi_{i}^{\mathrm{T}} \mathbf{x}_{s}\right)^{2}+\lambda P_{\omega}\left(\psi_{i}\right)\right)
$$

where $\lambda \geq 0$ is a chosen regularization parameter and $P_{\omega}\left(\psi_{i}\right)$ is the penalization term defined as

$$
P_{\omega}\left(\psi_{i}\right)=(1-\omega)\left\|\boldsymbol{\psi}_{i}\right\|_{2}^{2}+\omega\left\|\boldsymbol{\psi}_{i}\right\|_{1}=\sum_{j=1}^{3}\left((1-\omega)\left(\psi_{i}^{j}\right)^{2}+\omega\left|\psi_{i}^{j}\right|\right), \omega \in[0,1] .
$$

Note that for $\omega=0$ we get the so-called ridge regression and for $\omega=1$ we have the lasso estimator. It is clear that using this procedure, estimated coefficients depend on the choice of the regularization parameter $\lambda$. It is not known how to choose this parameter a priori, however, we may help ourselves with the technique of cross-validation. So we divide the fitting data period (say $[0, t]$ ) into $k$ folds of the same length and follow these steps:

1. One fold is selected as out-of-sample period, the rest as in-sample.

2. For a given $\lambda \geq 0$, we get the regularized elastic-net estimates of the model coefficients using the in-sample data.

3. Using the coefficients from the previous step, we produce model predicted values of the dependent variable in the out-of-sample fold and calculate the mean squared error (MSE).

4. Steps $1-3$ are repeated for all folds so that finally we get the MSE over all out-ofsample folds for a given $\lambda$. The division to folds may be also randomly rearranged and the process repeated, so the MSE is then also calculated over these repetitions.

5. We reiterate steps $1-4$ for a chosen range of regularization parameters $\left[\lambda_{\min }, \lambda_{\max }\right]$. Range is selected in such a way that we set $\lambda_{\max }=\max \left\{\lambda ; \exists j \in\{1,2,3\}: \quad \psi_{i}^{j}(\lambda) \neq 0, \lambda \geq 0\right\}$ and then $\lambda_{\min }=0.0001 \lambda_{\max }$.

Hence we have a function $\operatorname{MSE}^{\mathrm{CV}}(\lambda)$ that gives the cross-validation MSE over all (outof-sample) folds (and division repetitions) for a given $\lambda$. Then we choose the "best" $\lambda$ as

$$
\lambda^{*}=\arg \min _{\lambda \geq 0} \operatorname{MSE}^{\mathrm{cv}}(\lambda)
$$

Finally, we estimate the coefficients $\kappa_{i}$ and $\psi_{i}$ corresponding to $\lambda^{*}$ using the whole data period $[0, t]$ and these are the coefficients we use at time iteration $t$ to produce the estimate of $\theta_{t, h}{ }^{i}, i \in R$. The procedure runs similarly for the rolling window version, but we take $[t-\tau, t]$ instead of $[0, t]$. For a comparison we also propose an alternative way of $\lambda$ selection, concretely

$$
\tilde{\lambda}^{*}=\max \left\{\lambda ; \forall j \in\{1,2,3\}: \psi_{i}^{j}(\lambda) \neq 0, \lambda \geq 0\right\}
$$

i.e. maximal penalization such that all predictors have still some non-zero impact. Note that the whole procedure is conducted at each step when the prediction of jump model 
parameters and FX rates is produced. Although (15) and (16) are (for brevity) formulated only for the estimation of jump model regression coefficients of (8), the same procedure is used for the Gauss model and for the direct forecast (14).

Lastly, let us note that the AR model (9) coefficients are estimated at each step (with the available information) using ordinary least squares with no regularization or crossvalidation.

\subsection{Forecast evaluation criteria}

We employ several measures to evaluate forecast accuracy and compare different prediction methods, namely forecast of the jump model framework and the benchmark models. First, there is a simple out-of-sample MSE calculated as

$$
\operatorname{MSE}^{\mathrm{oos}}=\frac{h}{T-\tau} \sum_{t=\tau}^{T-h}\left(\Delta \hat{s}_{t+h \mid t}-\Delta s_{t+h \mid t}\right)^{2}=\frac{h}{T-\tau} \sum_{t=\tau}^{T-h}\left(\hat{s}_{t+h}-s_{t+h}\right)^{2}
$$

for an out-of-sample (OOS) period $[\tau, T]$. Standard benchmark for FX rates forecasting is the random walk $(R W)$ model (without drift), i.e. no-change forecast $\hat{s}_{t+h}^{\mathrm{rw}}=s_{t}, t=\tau, \ldots, T-h$. This yields MSE of the form $\mathrm{MSE}_{\mathrm{rw}}^{\text {oos }}=\frac{h}{T-\tau} \sum_{t=\tau}^{T-h}\left(s_{t+h}-S_{t}\right)^{2}$. Thus we may calculate the OOS coefficient of determination defined by

$$
R_{\mathrm{oos}}^{2}=1-\frac{\mathrm{MSE}^{\mathrm{oos}}}{\mathrm{MSE}_{\mathrm{rw}}^{\mathrm{oos}}},
$$

so we see that values greater than zero indicate more accurate prediction than the RW model, and vice versa. Furthermore, we also state other measures of accuracy such as the percentage of correct forecast sign and the mean bet return (per forecast period). The former is clear, the latter is given by

$$
\frac{h}{T-\tau} \sum_{t=\tau}^{T-h} \operatorname{sign}\left(\Delta \hat{s}_{t+h \mid t}\right) \frac{S_{t+h}-S_{t}}{S_{t}}
$$

which shall be compared with the mean FX rate return $\frac{h}{T-\tau} \sum_{t=\tau}^{T-h} \frac{S_{t+h}-S_{t}}{S_{t}}$. We also make use of a paired t-test to test against the alternative that the mean bet return is greater than the mean return of FX rate (data comprise of individual observations of the corresponding simple returns with/without the predicted sign adjustment sampled with frequency $h$ over the whole OOS test period).

Apart from these common measures of accuracy, we also use statistical tests to decide whether a forecast beats the RW benchmark or not. Firstly, we work with the Evans-Lyons (EL) test that is mentioned in Evans and Lyons (2005) for instance. The idea of the test is straightforward. It is based on regression of the forecasts on the realized values of returns, i.e.

$$
\Delta \hat{s}_{t+h \mid t}=\zeta+\varphi \Delta s_{t+h \mid t}+\varepsilon_{t}
$$

The null hypothesis is that $s_{t}$ follows a RW, so the correct forecast is $\Delta \hat{s}_{t+h \mid t}=0$. Therefore under the null, forecast $\Delta \hat{s}_{t+h \mid t}$ shall differ from zero only due to sampling error and 
should not be correlated with $\Delta s_{t^{\prime}+h \mid t}$ since it depends only on the information up to time $t$. This means we test for the significance of the $\varphi$ coefficient. $^{8}$

The second test we apply is the so-called Clark-West $(\mathrm{CW})$ test, see Clark and West (2006). Essentially, CW tests the same hypothesis as the EL test, namely that the returns follow a RW (and therefore have no connection with the fundamentals). In more detail, they test the null $\boldsymbol{\beta}=0$ for the model $\Delta s_{t+h \mid t}=\boldsymbol{\beta}^{\mathrm{T}} \mathbf{x}_{t}+\varepsilon_{t}$. They calculate out-of-sample MSE for the RW model, denote it

$$
\hat{\sigma}_{1}^{2}=\frac{h}{T-\tau} \sum_{t=\tau}^{T-h}\left(\Delta s_{t+h \mid t}\right)^{2}
$$

and its counterpart for the model using predictors

$$
\hat{\sigma}_{2}^{2}=\frac{h}{T-\tau} \sum_{t=\tau}^{T-h}\left(\Delta s_{t+h \mid t}-\hat{\boldsymbol{\beta}}_{t}^{\mathrm{T}} \mathbf{x}_{t}\right)^{2},
$$

where $\hat{\boldsymbol{\beta}}_{t}$ are the estimates of coefficients using information up to time $t$ (rolling or recursive estimation). Defining $\hat{\digamma}=\hat{\sigma}_{1}^{2}-\left(\hat{\sigma}_{2}^{2}-\frac{h}{T-\tau} \sum_{t=\tau}^{T-h}\left(\hat{\boldsymbol{\beta}}_{t}^{\mathrm{T}} \mathbf{x}_{t}\right)^{2}\right)$ and $\hat{V}=\frac{4 h}{T-\tau} \sum_{t=\tau}^{T-h}\left(\Delta s_{t+h \mid t} \hat{\boldsymbol{\beta}}_{t}^{\mathrm{T}} \mathbf{x}_{t}\right)^{2}$,
Clark and West show that

$$
\frac{\digamma \sqrt{T-\tau}}{\sqrt{h V}}
$$

has asymptotically standard normal distribution $\mathcal{N}(0,1)$ under the null hypothesis. ${ }^{9}$ In Clark and West (2006) there is more information about the test and its accuracy; also the case with serial correlation is treated there (overlapping forecast periods), although we do not need it since we have forecast horizon equal to the sampling period. Note that the test can by straightforwardly applied to the direct forecast case, for the jump models and the Gauss model benchmark we may consider that $x_{t}=\Delta \hat{s}_{t+h \mid t}$ and $\hat{\beta}_{t} \equiv 1$.

The last result connected with prediction evaluation we state is a simple trading test scheme. It consists of a basic betting on the FX rate returns according to the Kelly criterion ${ }^{10}$ using the predicted mean and $\mathrm{v}_{\max }$ ice of the jump model forecast framework. Now we explain what Kelly criterion bet means in essence. Let us assume we want to maximize expected logarithmic ${ }^{11}$ value of our portfolio in a bet on the FX rate return. To determine a fraction of our wealth $W$ we want to bet we solve

$$
\max _{c} \mathbb{E} \log \left(W\left(1+c \Delta s_{t+h \mid t}\right)\right)
$$

Using the Taylor expansion we write $\log \left(1+c \Delta s_{t+h \mid t}\right) \approx c \Delta s_{t+h \mid t}-\frac{\left(c \Delta s_{t+h \mid t}\right)^{2}}{2}$. Hence if we use this for the approximation of the objective function in (22) and take the derivative with respect to $c$ we get the (heuristically) optimal

$8 \quad$ Note that residuals of the EL test (auxiliary) model are auto-correlated by definition. Hence we use the well-known quasi-differencing technique to overcome this problem.

9 Variables without hat are the "true" variables whose estimates are given by the stated hat variables.

10 Introduced in Kelly (1956).

11 The logarithm is in the role of a utility function. 


$$
c^{*}=\frac{\mathbb{E} \Delta s_{t+h \mid t}}{\operatorname{var}\left(\Delta s_{t+h \mid t}\right)}=\frac{\mathcal{M}_{r}(\theta, h)}{\mathcal{V}_{r}(\theta, h)}
$$

where we have neglected $\left(\mathbb{E} \Delta s_{(t+h \mid t)}\right)^{2} \approx 0$. Recalling (10) and (12), if we have parameters of the jump model predicted, we know what ratio of our wealth we want to bet; analogously for the Gauss model benchmark using (13). Naturally, the trading test is not conducted for the direct forecast. Note that short bets are allowed and leverage might be used (these are realistic assumptions for the FX futures markets for instance). The bet wealth $W$ remains the same for each bet we make (so it is possible to go in debt - negative bankroll).

\section{Empirical Test Results}

\subsection{Used data}

As FX rate time series, we use spot FX rate data with a one hour granularity provided by a Swiss FX broker. The test is conducted on EUR/USD and USD/DKK currency pairs. ${ }^{12}$ For both pairs we have data for the period 01/2004-12/2013. Furthermore, we need the economic data for the calculation of predictors. All economic indicators are gathered in a monthly frequency. For price levels we use relative consumer price indices $(C P I)$ provided by the OECD statistics library. As the money supply we take the monetary aggregate $M 1$, again from the OECD data. As a proxy for the output we use the index of industrial production (IPI) since it is released on a monthly basis, source is the OECD. Potential output for the output gap is calculated using the one-sided Hodrick-Prescott filter, see Stock and Watson (1999). This maintains that predictors do not look to the future. Inflation is calculated as a yearly log-return of the CPI.

\subsection{Out-of-sample performance comparison}

Here we give results of our OOS test framework described in Subsection 2.4. Our main goal is to compare prediction using the jump models with the benchmarks of the direct forecast and the Gauss model prediction applying the criteria stated in Subsection 2.5. The test setting is following. We start the OOS test in the middle of the period for which we have data, i.e. $\tau=\frac{T}{2}$. This means we have a five-year-long OOS period 01/2009-12/2013. Our forecast horizon $h$ is set to one month while the time step $\Delta t$ for log-returns used to fit jump models is one hour. There are four versions of the results that differ in the type of the moving estimation (rolling or recursive) and in the way by which regularization parameter $\lambda$ is chosen, recall (17) and (18). The parameter of the elastic-net penalization term (16) is set to $\omega=0.5$. Number of folds in cross-validation is set to 6 and number of the cross-validation repetitions to 20 . Note that parameter forecasts produced by the AR model (9) are cut-offed by the 90 th percentile of the previously ${ }^{13}$ observed values since we want to avoid extremal predictions (and high volatility parameters may lead to such predictions since they escalate the influence of skewness parameters on the prediction).

12 The home currency is the second one stated for the pair.

13 Using no information from the future. 
Table 1 gives the performance results for EUR/USD, Table 2 for USD/DKK. Measures stated in the tables are as follows: \%CS is the percentage of correct forecast sign, $R_{\text {oos }}^{2}$ is defined in (20), MBR denotes the mean bet return introduced in (21), CW labels CW test $p$-value, EL corresponding EL test $p$-value, T denotes $p$-value of a paired $t$-test of the mean bet return significance, and finally TTR is an acronym for the (annualized) trade test return (of the simple Kelly bet trading scheme outlined in Subsection 2.5). Note that variables $R_{\text {oos }}^{2}$, MBR, and TTR are given in percent (i.e. multiplied by 100). MBR can be compared to the mean FX rate (per period) return denoted MFXR (also given in per cent) and stated below each of the tables.

Considering EUR/USD results, we see that jump models perform fairly better. In majority of the cases, statistical tests show they are significantly better than the RW model. For the direct forecast and the Gauss model, this happens to be true only for the case where $\tilde{\lambda}^{*}$ is selected by (18), especially for the rolling window regression. TTR is always higher for the jump models than for the continuous Gauss model. However, we may also see that good $R_{\text {oos }}^{2}$ does not always correspond with a good MBR or \%CS. MBR (or, more accurately, the true mean estimated by this sample mean) seems to be significantly higher than MFXR only for the rolling window estimation with $\tilde{\lambda}^{*}$ gained by (18).

Table 1 | OOS Test Results for EUR/USD

\begin{tabular}{|c|c|c|c|c|c|c|c|c|}
\hline Test Type & Model & $\% \mathrm{CS}$ & $R_{\text {oos }}^{2}$ & MBR & CW & EL & $\mathbf{T}$ & TTR \\
\hline \multirow{4}{*}{$\begin{array}{c}\text { Recursive } \\
\lambda^{*}\end{array}$} & NIG & 57.89 & 6.63 & 0.55 & $0.053^{*}$ & $0.028^{* *}$ & 0.199 & 44.63 \\
\hline & MXN & 52.63 & 9.25 & 0.29 & $0.039^{* *}$ & $0.014^{* *}$ & 0.312 & 51.48 \\
\hline & Gauss & 49.12 & -0.54 & -0.21 & 0.827 & 0.795 & 0.579 & 8.34 \\
\hline & Direct & 45.61 & 0.13 & -0.01 & 0.573 & 0.558 & 0.470 & NA \\
\hline \multirow{4}{*}{$\begin{array}{c}\text { Recursive } \\
\tilde{\lambda}^{*}\end{array}$} & NIG & 54.38 & 9.82 & 0.77 & $0.039^{* *}$ & $0.015^{* *}$ & 0.124 & 58.92 \\
\hline & MXN & 49.12 & 8.99 & 0.07 & $0.051^{*}$ & $0.013^{* *}$ & 0.422 & 52.40 \\
\hline & Gauss & 59.64 & 4.02 & 0.84 & $0.044^{* *}$ & $0.007^{* * *}$ & 0.105 & 26.87 \\
\hline & Direct & 54.38 & 3.77 & 0.66 & $0.095^{*}$ & $0.069^{*}$ & 0.159 & NA \\
\hline \multirow{4}{*}{$\begin{array}{c}\text { Rolling } \\
\lambda^{*}\end{array}$} & NIG & 59.64 & 4.42 & 0.63 & 0.122 & $0.088^{*}$ & 0.169 & 37.67 \\
\hline & MXN & 57.89 & 11.88 & 0.60 & $0.019^{* *}$ & $0.004^{* * *}$ & 0.178 & 57.33 \\
\hline & Gauss & 45.61 & -9.47 & -0.01 & 0.998 & 0.980 & 0.470 & -5.47 \\
\hline & Direct & 42.10 & -14.76 & 0.22 & 0.831 & 0.832 & 0.346 & NA \\
\hline \multirow{4}{*}{$\begin{array}{l}\text { Rolling } \\
\tilde{\lambda}^{*}\end{array}$} & NIG & 54.38 & 10.48 & 0.90 & $0.030^{* *}$ & $0.010^{* * *}$ & $0.090^{*}$ & 52.72 \\
\hline & MXN & 50.87 & 8.43 & 0.39 & $0.059^{*}$ & $0.026^{* *}$ & 0.264 & 45.40 \\
\hline & Gauss & 61.40 & 5.22 & 1.10 & $0.021^{* *}$ & $0.006^{* * *}$ & $0.052^{*}$ & 33.25 \\
\hline & Direct & 61.40 & 7.17 & 1.31 & $0.008^{* * *}$ & $0.004^{* * *}$ & $0.026^{* *}$ & NA \\
\hline
\end{tabular}

Note: Numbers' Superscripts $*^{* *},{ }^{* *}$, and ${ }^{*}$ denote rejection of the null hypothesis at the level of significance $0.01,0.05$, and 0.1 , respectively; MFXR $=-0.06$

Source: own computation 
For USD/DKK we observe that $R_{\text {oos }}^{2}$ of the jump models are lower than in the EUR/USD case. Even though statistical tests are not so persuasive for this currency, we see that jump models still perform somewhat better than the direct forecast or the Gauss model (no setting rejects the RW null for any of the benchmark models). The Meixner model works well for almost all of the test settings, but delivers a rather bad performance for the rolling window regression with $\lambda^{*}$ selected by Equation 17, though this setting is the worst for the other models as well.

Example of the NIG model prediction and the direct forecast of log-returns is depicted in Figure 3. The MXN model forecast and the direct forecast are compared in Figure 4. Prediction of the NIG model parameters is illustrated in Figure 5, analogous for the MXN model in Figure 6.

Table 2 | OOS Test Results for USD/DKK

\begin{tabular}{|c|c|c|c|c|c|c|c|c|}
\hline Test Type & Model & $\%$ CS & $\boldsymbol{R}^{2}$ oos & MBR & CW & EL & $\mathbf{T}$ & TTR \\
\hline \multirow{4}{*}{$\begin{array}{c}\text { Recursive } \\
\lambda^{*}\end{array}$} & NIG & 54.38 & 3.01 & 0.54 & 0.163 & 0.200 & 0.328 & 32.49 \\
\hline & MXN & 63.15 & 6.63 & 1.24 & $0.033^{* *}$ & $0.050^{* *}$ & $0.079^{*}$ & 42.56 \\
\hline & Gauss & 45.61 & -0.72 & 0.09 & 0.786 & 0.817 & 0.564 & 6.53 \\
\hline & Direct & 45.61 & 0.41 & 0.13 & 0.329 & 0.429 & 0.545 & NA \\
\hline \multirow{4}{*}{$\begin{array}{c}\text { Recursive } \\
\tilde{\lambda}^{*}\end{array}$} & NIG & 61.40 & 4.78 & 0.78 & 0.162 & $0.085^{*}$ & 0.222 & 50.27 \\
\hline & MXN & 54.38 & 8.18 & 0.90 & $0.030^{* *}$ & $0.026^{* *}$ & 0.175 & 49.87 \\
\hline & Gauss & 61.40 & 2.37 & 0.81 & 0.157 & 0.105 & 0.209 & 18.49 \\
\hline & Direct & 59.64 & 2.00 & 0.72 & 0.283 & 0.255 & 0.245 & NA \\
\hline \multirow{4}{*}{$\begin{array}{c}\text { Rolling } \\
\lambda^{*}\end{array}$} & NIG & 52.63 & 0.76 & -0.08 & 0.346 & 0.413 & 0.656 & 27.91 \\
\hline & MXN & 57.89 & -18.74 & 0.74 & 0.525 & 0.499 & 0.238 & -65.33 \\
\hline & Gauss & 52.63 & -1.47 & 0.12 & 0.475 & 0.508 & 0.552 & 27.48 \\
\hline & Direct & 54.38 & -2.84 & 0.63 & 0.371 & 0.443 & 0.285 & NA \\
\hline \multirow{4}{*}{$\begin{array}{l}\text { Rolling } \\
\tilde{\lambda}^{*}\end{array}$} & NIG & 59.64 & 5.90 & 0.71 & 0.142 & $0.058^{*}$ & 0.250 & 50.06 \\
\hline & MXN & 59.64 & 8.15 & 1.24 & $0.046^{* *}$ & $0.023^{* *}$ & $0.079^{*}$ & 45.57 \\
\hline & Gauss & 61.40 & 2.49 & 0.68 & 0.173 & 0.144 & 0.265 & 20.61 \\
\hline & Direct & 61.40 & 3.67 & 0.74 & 0.114 & 0.114 & 0.238 & NA \\
\hline
\end{tabular}

Note: Numbers' superscripts $* * * * *$, and ${ }^{*}$ denote rejection of the null hypothesis at the level of significance $0.01,0.05$, and 0.1 , respectively; MFXR $=0.22$

Source: own computation 
Figure 3 | Log-Returns Forecast Comparison - NIG versus Direct Forecast

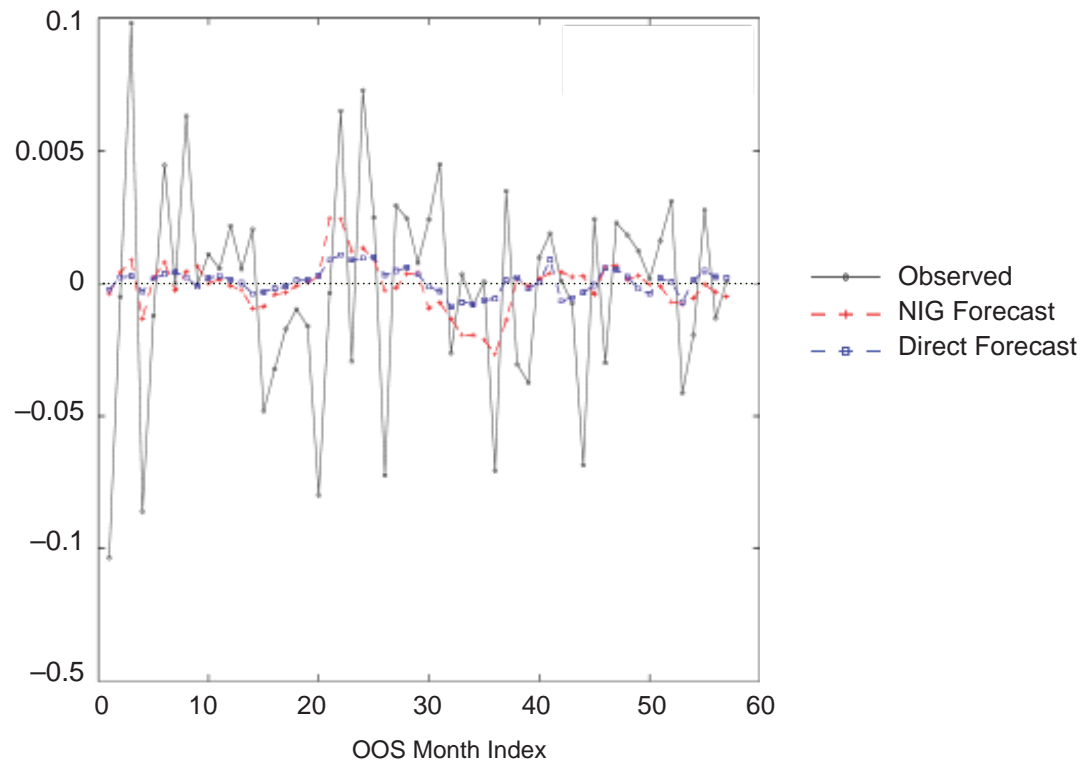

Note: EUR/USD rate, OOS period 01/2009-12/2013, rolling window estimation with $\tilde{\lambda}^{*}$ selected by (18) Source: own computation

Figure 4 | Log-Returns Forecast Comparison - MXN versus Direct Forecast

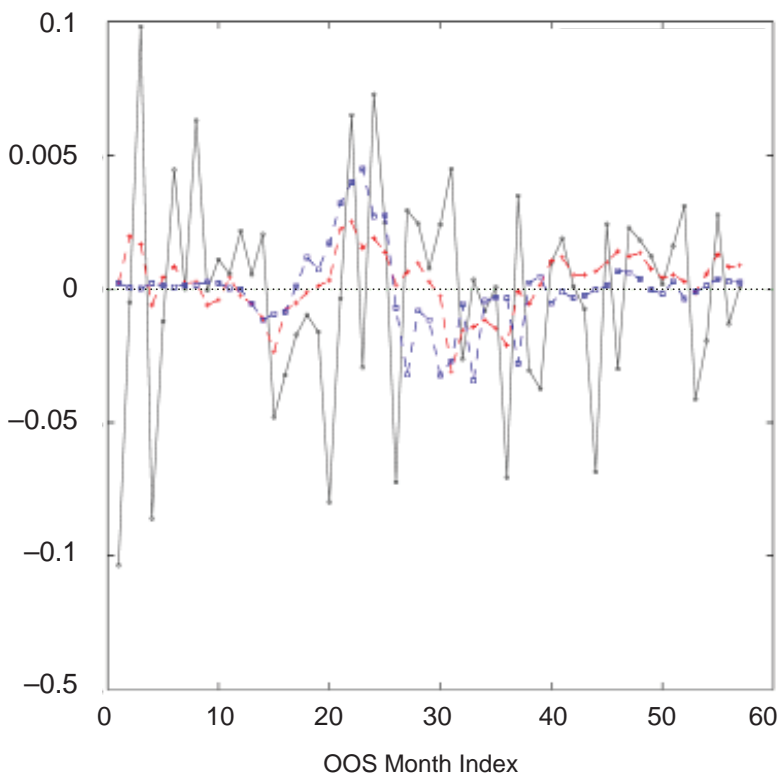

$\longrightarrow$ Observed

- + - Meixner Forecast

- $\#$ - Direct Forecast

Note: EUR/USD rate, OOS period 01/2009-12/2013, rolling window estimation with $\lambda^{*}$ selected by (17) Source: own computation 
Figure 5 | NIG Model Predicted (dashed, plus marker) versus Observed (solid, circle marker) Parameters
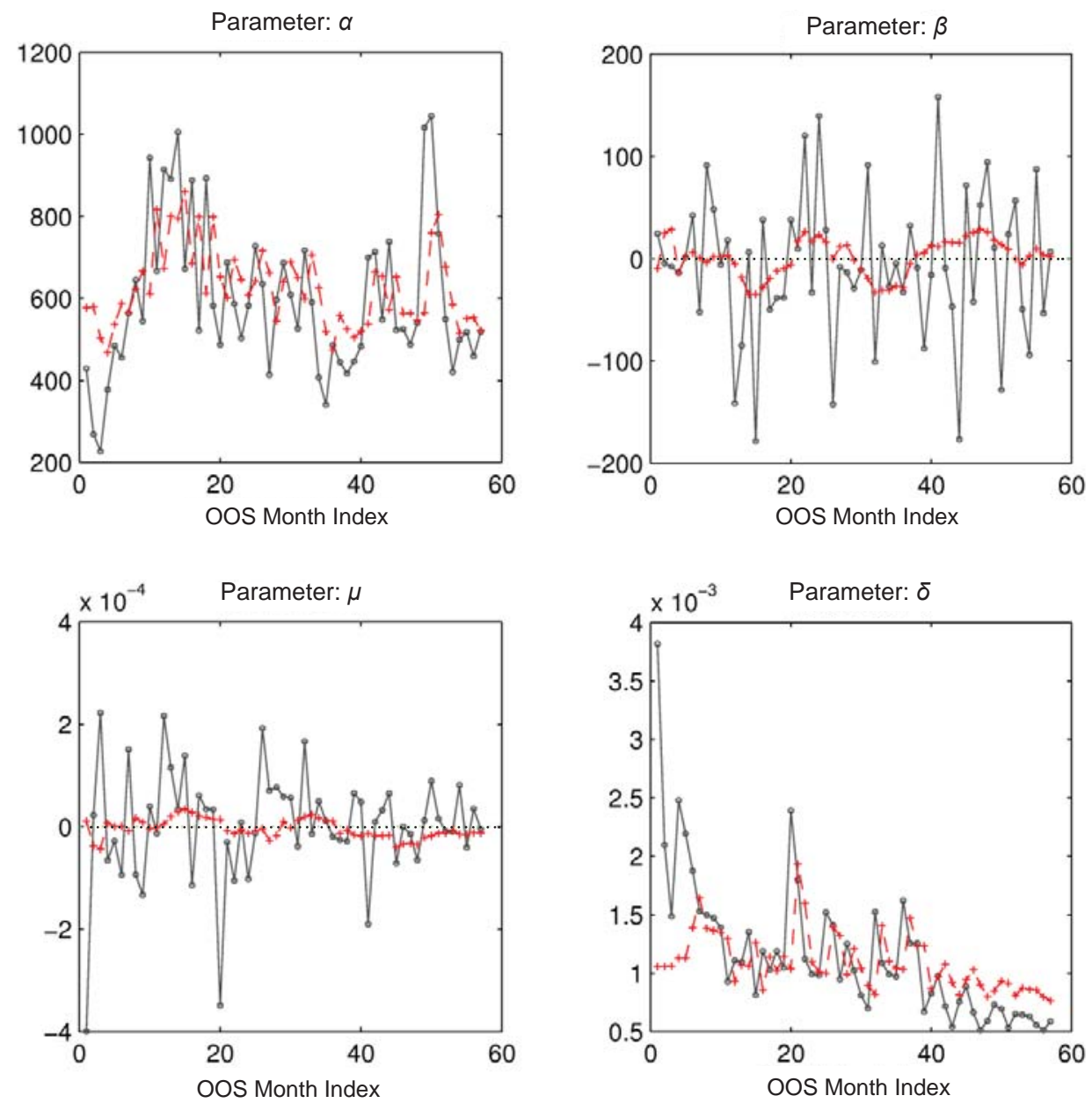

Note: EUR/USD rate, OOS period 01/2009-12/2013, rolling window estimation with $\tilde{\lambda}^{*}$ selected by (18) Source: own computation 

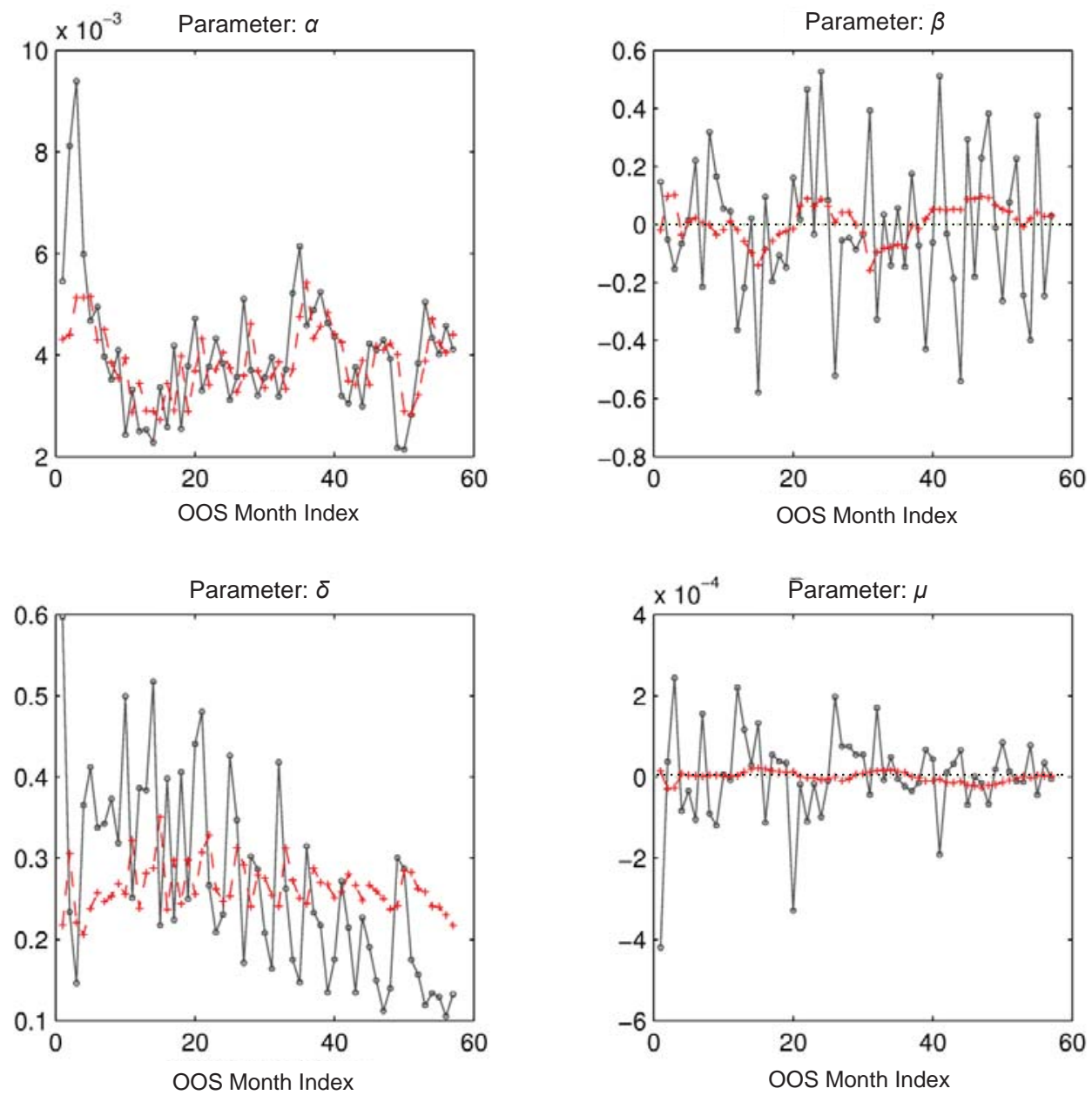

Note: EUR/USD rate, OOS period 01/2009-12/2013, rolling window estimation with $\lambda^{*}$ selected by (17) Source: own computation

\section{Concluding Remarks}

We showed a way how stochastic models based on jump processes can be combined with macroeconomic fundamentals in order to predict FX rates. The results suggest that although there is a sign of forecast accuracy improvement (over the direct forecast and the Gauss benchmark models), it is not unequivocal. We see that performance hinges on a chosen evaluation measure and a selected test setting. It is also difficult to find a robust prediction approach for different currency pairs since we observe that performance changes if we move from one pair to another, ceteris paribus. This unstability of results is a known fact (see Rossi (2013) for instance) and it underscores the problem of describing a robust connection 
between FX rates and macroeconomic variables. However, we hope we have exemplified a new approach to this problem that might be developed further and help in clarification of the exchange rate disconnect puzzle eventually. Generally, to choose the best modelling approach is a difficult question in essence. Perhaps it depends on a goal for which one uses the prediction. For example, if you are concerned with the direction of prediction only, then you should look on the percentage of correct forecast sign or MBR (compared with MFXR). If you also want to scale your bet on the FX rate, then TTR might be an appropriate criterion. If you want to compare the prediction with the RW model, then you may choose $R_{\mathrm{oos}}^{2}$ and the results of employed statistical tests. Either way, one could perhaps say that MXN model with the rolling estimation and minimal MSE $\lambda^{*}$ is the best choice for EUR/ USD, or that MXN model with the rolling estimation and the "least non-zero-all influence" $\tilde{\lambda}^{*}$ is the best alternative for USD/DKK.

There are multiple options of modification and extension of our research. First, different definitions and sets of macroeconomic fundamentals may be chosen (e.g. fundamentals based on interest rate parity or forward $F X$ rates). However, one has to realize that simple addition of predictors does not have to improve out-of-sample performance. Second, methods used for the prediction of jump model parameters may be altered. We use the fundamental linear model which is made more robust for OOS performance by regularization, but other choices such as non-linear models, panel models, or artificial neural networks may be considered. Likewise, a different (than AR) model may be used for the volatility parameters, although these parameters are less crucial for the forecast sign determination. Lastly, of course, tests might be conducted on different data sets (periods) and for other currencies. Indeed, longer data examples would be interesting, but it is not that simple to acquire a longer period of high-frequency ${ }^{14} \mathrm{FX}$ rate time series.

\section{References}

Abramowitz, M., Stegun, I. A. (1972). Handbook of Mathematical Functions with Formulas, Graphs, and Mathematical Tables. 9th printing. New York: Dover Books.

Barndorff-Nielsen, O. E. (1997). Normal Inverse Gaussian Distributions and Stochastic Volatility Modelling. Scandinavian Journal of Statistics, 24(1), 1-13. DOI: 10.1111/1467-9469. t01-1-00045.

Bates, D. S. (1996). Jumps and Stochastic Volatility: Exchange Rate Process Implicit in Deutsche Mark Options. The Review of Financial Studies, 9(1), 69-107. DOI: 10.1093/rfs/9.1.69.

Božović, M. (2008). The Role of Jumps in Foreign Exchange Rates. Universitat Pompeu Fabra Barcelona Working Paper.

Bruyn, R. D., Gupta, R., Stander, L. (2013). Testing the Monetary Model for Exchange Rate Determination in South Africa: Evidence from 101 Years of Data. Contemporary Economics, 7(1), 19-32. DOI: 10.5709/ce.1897-9254.71.

Bunčák, T. (2013). Jump Processes in Exchange Rates Modeling, in Proceedings of the 6th International Scientific Conference for Ph.D. Students and Young Scientists. Silesian University in Opava, 514-540.

Busch, T., Christensen, B. J., Nielsen, M. Ø. (2005). Forecasting Exchange Rate Volatility in the Presence of Jumps. Queen's Economics Department Working Paper No. 1187.

14 In order to calibrate jump models. 
Clark, T. E., West, K. D. (2006). Using Out-of-Sample Mean Squared Prediction Errors to Test the Martingale Difference Hypothesis. Journal of Econometrics, 135(1-2), 155-186. DOI: 10.1016/j.jeconom.2005.07.014.

Cont, R., Tankov, P. (2003). Financial Modelling with Jump Processes. London: Chapman \& Hall / CRC Press. DOI: 10.1201/9780203485217.

Engel, C., Mark, N. C., West, K. D. (2007). Exchange Rate Models Are Not as Bad as You Think, in NBER Macroeconomics Annual 2007. 22. National Bureau of Economic Research, Inc., pp. 381-441. DOI: 10.3386/w13318.

Evans, M. D. D., Lyons, R. K. (2005). Meese-Rogoff Redux: Micro-Based Exchange Rate Forecasting. American Economic Review, 95(2), 405-414.

DOI: $10.1257 / 000282805774669934$.

Jiang, G. J. (1998). Jump-Diffusion Model of Exchange Rate Dynamics: Estimation via Indirect Inference. University of Groningen - Working Paper.

Kelly, J. L. (1956). A New Interpretation of Information Rate. Bell System Technical Journal, 35(4), 917-926. DOI: 10.1002/j.1538-7305.1956.tb03809.x.

Li, J., Tsiakas, I., Wang, W. (2015). Predicting Exchange Rates Out of Sample: Can Economic Fundamentals Beat the Random Walk? Journal of Financial Econometrics, 13(2), 293-341. DOI: 10.1093/jjfinec/nbu007.

Maheu, J. M., McCurdy, T. H. (2008). Modeling Foreign Exchange Rates with Jumps, in Rapach, D. E., Wohar, M. E., eds., Forecasting in the Presence of Structural Breaks and Model Uncertainty. Emerald Group, pp. 449-475. DOI: 10.1016/s1574-8715(07)00212-6.

Meese, R. A., Rogoff, K. (1983). Empirical Exchange Rate Models of the Seventies: Do They Fit Out of Sample? Journal of International Economics, 14, pp. 3-24. DOI: 10.1016/0022-1996(83)90017-x.

Nekhili, R., Altay-Salih, A., Gençay, R. (2002). Exploring Exchange Rate Returns at Different Time Horizons. Physica A, 313, pp. 671-682. DOI: 10.1016/s0378-4371(02)00986-x.

Nirei, M., Sushko, V. (2011). Jumps in Foreign Exchange Rates and Stochastic Unwinding of Carry Trades. International Review of Economics \& Finance, 20(1), 110-127. DOI: 10.1016/j.iref.2010.07.010.

Rossi, B. (2013). Exchange Rate Predictability. Journal of Economic Literature, 51(4), 1063-1119. DOI: 10.1257/jel.51.4.1063.

Schoutens, W., Teugels, J. L. (1998). Lévy Processes, Polynomials and Martingales. Communications in Statistics - Stochastic Models, 14, pp. 335-349. DOI: 10.1080/15326349808807475.

Stock, J. H., Watson, M. W. (1999). Forecasting Inflation. Journal of Monetary Economics, 44(2), 293-335. DOI: 10.1016/s0304-3932(99)00027-6.

Zou, H., Hastie, T. (2005). Regularization and Variable Selection via the Elastic Net. Journal of the Royal Statistical Society, Series B, 67(2), 301-320.

DOI: 10.1111/j.1467-9868.2005.00503.x. 